\title{
Escores prognósticos à admissão e desfechos de pacientes em unidade de terapia intensiva
}

\author{
Prognostic scores for admission and outcomes of patients in a intensive care unit \\ Pontuaciones prognósticas en la admisión y salidas de pacientes en una unidad de \\ atención intensiva
}

Yonara Yasmim Ferreira Anjos ${ }^{1 *}$, Brenda Suelen Nascimento Vasconcelos ${ }^{1}$, Cleidinaldo Ribeiro de Goes Marques², Nathalia Vasconcelos Barroso Todt Aragão', Eduesley Santana Santos².

\section{RESUMO}

Objetivo: Objetivou identificar a associação entre os escores da admissão e desfechos desfavoráveis em pacientes de UTI. Métodos: Trata-se de análise transversal de uma coorte prospectiva. Foram utilizados os escores: Simplified Acute Physiology III (SAPS III), Sequential Organ Failure Assessment (SOFA), Nursing Activities Score (NAS) e Índice de comorbidade de Charlson (ICC). Foram coletados dados de pacientes com idade maior ou igual a 18 anos no período de outubro de 2018 a julho de 2019. A amostra foi composta por 100 pacientes, com idade média de $68 \pm 19$ anos. Os escores calculados na admissão foram: ICC (4 \pm 3$)$, SAPS III (28 \pm 12 ) e SOFA ( $3 \pm 3$ ) e no desfecho foram $2 \pm 4$ (SOFA) e $29 \pm 16$ (SAPS III). Resultados: A procedência prevalente foi emergência, com diagnóstico cardiovascular, antecedentes pessoais cirurgia prévia, HAS como comorbidade, suporte admissional noradrenalina e fentanil e dispositivo mais utilizado sonda vesical de demora. Conclusão: Pode-se evidenciar a associação de desfechos clínicos desfavoráveis ao paciente com escores assistências. Torna-se essencial sua utilização na prática clínica de modo a guiar a assistência prestada ao paciente crítico.

Palavras-chave: Unidade de terapia intensiva, Escala psicológica aguda simplificada, Prognóstico, Serviço hospitalar de admissão de pacientes, Assistência ao paciente.

\section{ABSTRACT}

Objective: The objective was to identify the association between admission scores and unfavorable outcomes in ICU patients. Methods: This is a cross-sectional analysis of a prospective cohort. Scores were used: Simplified Acute Physiology III (SAPS III), Sequential Organ Failure Assessment (SOFA), Nursing Activities Score (NAS) and Charlson's comorbidity index (ICC). Data were collected from patients aged 18 years or older from October 2018 to July 2019. The sample consisted of 100 patients, with an average age of $68 \pm 19$ years. The scores calculated at admission were: ICC (4 \pm 3$)$, SAPS III (28 \pm 12$)$ and SOFA ( $3 \pm 3)$ and at the outcome were $2 \pm 4$ (SOFA) and $29 \pm 16$ (SAPS III). Results: The prevalent source was emergency, with cardiovascular diagnosis, personal history of previous surgery, SAH as a comorbidity, noradrenaline and fentanyl admission support and the most used device for bladder catheter delay. Conclusion: The association of unfavorable clinical outcomes to the patient with assistance scores can be evidenced. Its use in clinical practice is essential in order to guide the care provided to critical patients.

Keywords: Intensive care unit, Simplified acute physiology score, Prognosis, Hospital service for admission of patients, Patient care.

\footnotetext{
${ }^{1}$ Universidade Tiradentes (UNIT), Aracaju - SE.

*E-mail: yonaraanjos@gmail.com

2 Universidade Federal de Sergipe (UFS), Aracaju - SE.
} 


\section{RESUMEN}

Objetivo: Su objetivo era identificar la asociación entre las puntuaciones de admisión y los resultados desfavorables en pacientes de la UCl. Métodos: Este es un análisis transversal de una cohorte prospectiva. Se utilizaron las puntuaciones: fisiología aguda simplificada III (SAPS III), evaluación secuencial de insuficiencia orgánica (SOFA), puntuación de actividades de enfermería (NAS) e índice de comorbilidad de Charlson (ICC). Los datos se obtuvieron de pacientes de 18 años o más en el período comprendido entre octubre de 2018 y julio de 2019. La muestra consistió en 100 pacientes, con una edad promedio de $68 \pm 19$ años. Los puntajes calculados al ingreso fueron: ICC $(4 \pm 3)$, SAPS III $(28 \pm 12)$ y SOFA $(3 \pm 3)$ y en el resultado fueron $2 \pm 4$ (SOFA) y $29 \pm 16$ (SAPS III). Resultados: la fuente prevalente fue la emergencia, con diagnóstico cardiovascular, antecedentes personales de cirugía previa, HSA como comorbilidad, soporte de admisión de noradrenalina y fentanilo y el dispositivo más utilizado para el retraso del catéter vesical. Conclusión: Se puede evidenciar la asociación de resultados clínicos desfavorables para el paciente con puntajes de asistencia. Su uso en la práctica clínica es esencial para guiar la atención brindada a los pacientes críticos.

Palabras clave: Unidad de cuidados intensivos, Puntuación fisiológica simplificada aguda, Pronóstico, Servicio hospitalario de admisión de pacientes, Atención al paciente.

\section{INTRODUÇÃO}

A Unidade de Terapia Intensiva (UTI) é um local destinado a pacientes potencialmente graves que necessitam de monitorização contínua e de cuidados da equipe multiprofissional de forma integral, onde se concentram grandes recursos tecnológicos. Para tanto, surgiu devido à necessidade de prestar atendimento a pacientes em estado crítico, mas recuperáveis. Deste modo, a Organização Mundial da Saúde (OMS) preconiza que $7 \%$ a $10 \%$ do total dos leitos de hospitais cadastrados no Cadastro Nacional de Estabelecimentos de Saúde (CNES) sejam destinados à UTI (BRASIL, 2013; CALDEIRA VMH, et al., 2010; PASSOS SSS, et al., 2015).

O setor é marcado por uma heterogeneidade no perfil de pacientes admitidos e em algumas situações essa diferença impacta no tempo de internação e rotatividade na ocupação dos leitos. Não somente, a seleção de pacientes para serem admitidos é um assunto que há divergência relacionada ao consenso de gravidade e indicação de tratamento na UTI entre os profissionais da equipe para ocupação das vagas (CALDEIRA $\mathrm{VMH}$, et al., 2010).

Nesta perspectiva, faz-se necessário que a equipe multiprofissional tenha conhecimento do perfil destes pacientes para traçar os cuidados. Uma possibilidade de melhoria evidenciada é a utilização de escores assistenciais, os quais podem auxiliar nas decisões e tratamentos necessários, reduzindo a mortalidade e detectando riscos de forma precoce (RODRIGUEZ AH, et al., 2016).

O primeiro índice prognóstico criado foi o Acute Physiology and Chronic Health Evaluation (APACHE), e também na década de 1980 o Simplified Acute Physiology Score (SAPS) foi elaborado, porém apresentava limitações, sendo aprimorado até 2002 com o lançamento de sua terceira versão, que mostrou-se eficaz (BUENO HL, BIATTO JFP, 2015). A multiplicidade de comorbidades dos pacientes admitidos na UTI favoreceu a criação do Índice de Comorbidade de Charlson (ICC) (MARTINS M, 2010). O escore SOFA (Sequential Organ Failure Assessment) é utilizado como medida de avaliação da possibilidade desta disfunção orgânica e direciona para a conduta necessária (HISSA PNG, et al., 2013).

A utilização dos índices prognósticos está sendo disseminada nas UTI's revelando parâmetros de qualidade e eficácia da assistência prestada, podendo fazer estratificação da gravidade dos pacientes e seu prognóstico, e analisar a resposta à conduta terapêutica (SILVA LM, 2014). Contudo, ainda é baixo o número de pesquisas científicas abordando a temática. Diante disso, esse trabalho tem como objetivo identificar a associação entre os escores SAPSIII, SOFA e ICC, da admissão e desfechos desfavoráveis em pacientes de UTI particular de Aracaju/SE. 


\section{MÉTODOS}

Trata-se de uma análise transversal de uma coorte prospectiva intitulada "Caracterização clínica, perfil de gravidade e desfechos de pacientes em unidades de terapia intensiva". Este estudo foi conduzido em uma UTI particular localizada em uma cidade do estado de Sergipe, que divide o seu atendimento aos pacientes clínicos (20 leitos) e cirúrgico (5 leitos) (durante o período da coleta de dados).

A amostra foi por conveniência e não probabilística, com participantes de idade maior ou igual à 18 anos, admitidos na UTI, com tempo de permanência mínima de 24 horas. Os pacientes que evoluíram para óbito ou transferência intra e inter-hospitalar, durante as primeiras 24 horas, foram excluídos do estudo.

A coleta foi feita via prontuário eletrônico do próprio hospital que utiliza o sistema Tasy (onde estão reunidas todas as informações sobre o paciente, procedimentos realizados, bem como prescrições), entre os meses de outubro de 2018 a julho de 2019, em turnos e horários determinados pela instituição, sendo quatro dias da semana no turno vespertino.

$\mathrm{O}$ registro foi feito em instrumento criado pelos pesquisadores e os pacientes foram acompanhados durante o momento da internação na UTI até o sétimo dia de internação e no dia do desfecho. Após a saída da UTI, os pacientes foram acompanhados quanto aos desfechos até sua saída do hospital por alta, transferência ou óbito.

Foi utilizado instrumento de coleta de dados elaborado para o projeto contendo informações para caracterização clínica e demográfica, avaliação da carga de trabalho pelo Nursing Activities Score (NAS), prognóstico pelo SAPS III, disfunção pelo SOFA, comorbidades através do ICC, registro de parâmetros vitais, balanço hídrico $(\mathrm{BH})$ e exames laboratoriais.

Para esse trabalho foi utilizado o SAPS III, que possui 20 variáveis que estão divididas de fácil utilização, aplicado no momento da admissão e alta do paciente; SOFA, que atribui pontuação de 1 a 4 para a gravidade da disfunção de órgãos, aplicado a partir do segundo dia de internação na UTI e ICC, aplicado na admissão do paciente, levando em conta 19 possíveis condições clínicas sendo atribuído a estas, peso de um a seis, quanto maior o número, uma comorbidade mais grave e posteriormente o índice é ajustado a idade do paciente (SILVA JÚNIOR JM, 2010; KEEGAN MT e SOARES M, 2016; AUSTIN SR, et al., 2015).

Quanto a análise de dados, foram postos em tabelas no programa Excel 2010 e submetido à análise estatística no programa Statistical Package for the Social Sciences (SPSS) versão 25.0. As análises incluíram estatísticas demográficas da amostra e descritiva para as características clínicas. As variáveis categóricas são apresentadas sob a forma de frequências absolutas e relativas e as contínuas sob a forma de média e desvio padrão ou mediana e intervalo interquartílico. Para análise de associação dos escores com os desfechos foi utilizado o teste qui-quadrado ou exato de Fisher, para as variáveis categóricas, e o teste tStudent independente, para variáveis contínuas. O nível de significância utilizado foi de $p<0,05$.

Este estudo fez parte de um projeto do tipo guarda-chuva, aprovado pelo Comitê de Ética em Pesquisa da Universidade Federal de Sergipe (parecer $n^{\circ}$ 2.830.187) seguindo as recomendações da Resolução 466/12.

\section{RESULTADOS}

No presente trabalho, analisou-se os escores de forma rigorosa, onde foram apresentadas em tabelas os resultados obtidos. A amostra por conveniência foi composta por 100 pacientes, sem predominância de sexo, com idade média de $68 \pm 19$ anos, procedentes da Emergência (74\%), com disfunção cardíaca (31\%), histórico de cirurgia prévia (44\%) e portadores de HAS (58\%) em sua grande maioria.

Levando em conta o suporte admissional na UTI, houve predomínio do suporte farmacológico com Noradrenalina (14\%), Fentanil (14\%) e Dormonid (13\%) e suporte fisiológico por dispositivos invasivos por Sonda Vesical de Demora (35\%). Os escores calculados na admissão foram: ICC, SAPSIII e SOFA, e obtiveram a seguinte média respectivamente, $4 \pm 3,28 \pm 12$ e $3 \pm 3$. A média dos escores aplicados no desfecho $2 \pm 4$ (SOFA) e 29 \pm 16 (SAPS III). A Tabela 1 traz as características clínicas e demográficas dos pacientes. 
Tabela 1 - Características clínicas e sociodemográficas dos pacientes do estudo, Brasil, 2019.

\begin{tabular}{|c|c|c|}
\hline Variáveis & $\mathbf{n}$ & $\%$ \\
\hline Sexo, masculino, n (\%) & 50 & 50 \\
\hline Idade, média anos $\pm \mathrm{DP}$ & $68 \pm 19$ & - \\
\hline Peso, média \pm DP & $71 \pm 16$ & - \\
\hline Altura, mediana, quartis & $1,63[1,42-1,86]$ & - \\
\hline Índice de massa corpórea, média \pm DP & $27 \pm 6$ & - \\
\hline \multicolumn{3}{|l|}{ Procedência } \\
\hline Emergência, n (\%) & 74 & 74 \\
\hline Centro Cirúrgico, n (\%) & 18 & 18 \\
\hline Internamento, n (\%) & 8 & 8 \\
\hline \multicolumn{3}{|l|}{ Diagnóstico por sistema } \\
\hline Cardiovascular, n (\%) & 29 & 31 \\
\hline Respiratório, n (\%) & 19 & 19 \\
\hline Infeccioso, n (\%) & 13 & 13 \\
\hline Neurológico, n (\%) & 12 & 12 \\
\hline Digestório, n (\%) & 11 & 11 \\
\hline \multicolumn{3}{|l|}{ Antecedentes pessoais } \\
\hline Cirurgia prévia, $\mathrm{n}(\%)$ & 44 & 44 \\
\hline Infarto Agudo do Miocárdio prévio, n (\%) & 15 & 15 \\
\hline Tabagista prévio, n (\%) & 12 & 12 \\
\hline Acidente Vascular Encefálico prévio, n (\%) & 9 & 9 \\
\hline Creatinina basal $>1,5$ & 9 & 9 \\
\hline \multicolumn{3}{|l|}{ Comorbidades } \\
\hline Hipertensão Arterial Sistêmica, n (\%) & 58 & 58 \\
\hline Dislipidemia, n (\%) & 33 & 33 \\
\hline Diabete Mellitus, n (\%) & 30 & 30 \\
\hline Arritmia, n (\%) & 15 & 15 \\
\hline Doença Pulmonar Obstrutiva Crônica, n (\%) & 11 & 11 \\
\hline Hipotireoidismo, n (\%) & 11 & 11 \\
\hline Insuficiência Cardíaca, n (\%) & 10 & 10 \\
\hline \multicolumn{3}{|l|}{ Suporte admissional } \\
\hline Noradrenalina, $\mathrm{n}(\%)$ & 14 & 14 \\
\hline Fentanil, n (\%) & 14 & 14 \\
\hline Dormonid, n (\%) & 13 & 13 \\
\hline \multicolumn{3}{|l|}{ Dispositivos em uso } \\
\hline Sonda Vesical de Demora, n (\%) & 35 & 35 \\
\hline Cateter Venoso Central, n (\%) & 29 & 29 \\
\hline Sonda nasoenteral, n (\%) & 15 & 15 \\
\hline Tubo orotraqueal, n (\%) & 15 & 15 \\
\hline Dreno, n (\%) & 6 & 6 \\
\hline Sonda nasogástrica, n (\%) & 6 & 6 \\
\hline \multicolumn{3}{|l|}{ Escores admissionais } \\
\hline ICC, média \pm DP & $4 \pm 3$ & - \\
\hline SAPS III, média \pm DP & $28 \pm 12$ & - \\
\hline SOFA 24 h, média \pm DP & $3 \pm 3$ & - \\
\hline \multicolumn{3}{|l|}{ Escores no desfecho } \\
\hline SOFA, média \pm DP & $2 \pm 4$ & - \\
\hline SAPS III, média \pm DP & $29 \pm 16$ & - \\
\hline
\end{tabular}

Legenda: DP - desvio padrão

Fonte: Anjos YYF, et al., 2020. 
Dentre os desfechos dos pacientes (Tabela 2), destaca-se a infecção (47\%), com foco pulmonar tendo sua maior porcentagem (43\%), sendo seguida de outros focos como urinário (19\%) e de corrente sanguínea (13\%). O segundo maior desfecho é VM maior que 48 horas (37\%), seguido de Lesão Renal Aguda (LRA) (29\%), Lesão por Pressão (LPP) (24\%), entre outros. O IAM, obteve apenas $9 \%$ dos desfechos, ficando assim com a menor porcentagem. Porém, vale ressaltar que mesmo estando em último na tabela, possui tamanha importância em ser evitado, igualmente aos demais desfechos.

Tabela 2 - Desfechos dos pacientes, Brasil, 2019.

\begin{tabular}{lcc}
\hline Desfechos & $\mathbf{n ~ ( 1 0 0 )}$ & $\%$ \\
\hline Infecção, n (\%) & 47 & 47 \\
Foco pulmonar, n (\%) & 20 & 43 \\
Outro foco, n (\%) & 12 & 26 \\
Foco urinário, n (\%) & 9 & 19 \\
Foco de corrente sanguínea, n (\%) & 6 & 13 \\
\hline VM> 48h, n (\%) & 37 & 37 \\
LRA, n (\%) & 29 & 29 \\
LPP, n (\%) & 24 & 24 \\
Morte, n (\%) & 27 & 27 \\
Diálise, n (\%) & 19 & 19 \\
TIH, dias, mediana, quartis & $16[3-110]$ & - \\
TUTI, dias, mediana, quartis & $8[2-104]$ & - \\
\hline Reinternação, n (\%) & 14 & 14 \\
AVE, n (\%) & 10 & 10 \\
IAM, n (\%) & 9 & 9 \\
\hline
\end{tabular}

Legenda: VM> 48h - Ventilação Mecânica por mais de 48 horas; LRA- Lesão Real Aguda; LPP- Lesão por Pressão; TIH - tempo de internação hospitalar; TUTI - tempo de internação em UTI; AVE - Acidente Vascular Encefálico; IAM - Infarto Agudo do Miocárdio.

Fonte: Anjos YYF, et al., 2020.

Os escores prognósticos serviram de base para avaliação da assistência prestada aos pacientes admitidos na UTI. Quanto ao SAPS III, foi possível separar os pacientes em dois grupos, apresentando maior prevalência os que se encaixaram no SAPS $I I \geq 28(n=53)$. Levando em consideração este grupo, o desfecho de infecção apresentou maior porcentagem (66\%), seguido de VM>48h $(47,2 \%)$, LRA, LPP e óbito (32,1\%), Diálise $(18,9 \%)$. Analisando a associação do SAPS III com os desfechos, houve significância estatística com infecção $(p=0,014)$, TIH $(p=0,008)$, VM> 48h $(p=0,025)$, LPP $(p=0,045)$ e TUTI $(p=0,028)$. A Tabela 3 mostra a análise da associação dos desfechos dos pacientes com SAPSIII.

Tabela 3 - Análise da associação dos desfechos dos pacientes com SAPS III.

\begin{tabular}{|c|c|c|c|}
\hline Desfechos & $\begin{array}{c}\text { SAPS III }<28 \\
(n=47)\end{array}$ & $\begin{array}{c}\text { SAPS III } \geq 28 \\
(n=53)\end{array}$ & $\begin{array}{c}\text { p valor } \\
(0,05)\end{array}$ \\
\hline Infecção, n (\%) & $16(34 \%)$ & $31(66 \%)$ & $0,014^{*}$ \\
\hline $\mathrm{TIH}$, dias, média $\pm \mathrm{DP}$ & $18 \pm 16$ & $27 \pm 23$ & $0,008^{*}$ \\
\hline $\mathrm{VM}>48 \mathrm{~h}, \mathrm{n}(\%)$ & $12(25,5 \%)$ & $25(47,2 \%)$ & $0,025^{*}$ \\
\hline Morte, n (\%) & $10(21,3 \%)$ & $17(32,1 \%)$ & 0,225 \\
\hline LPP, n (\%) & $7(14,9 \%)$ & $17(32,1 \%)$ & $0,045^{*}$ \\
\hline TUTI, dias, média \pm DP & $9 \pm 11$ & $16 \pm 16$ & $0,028^{*}$ \\
\hline Reinternação, n (\%) & $5(10,6 \%)$ & $9(17 \%)$ & 0,362 \\
\hline AVE, n (\%) & $2(4,3 \%)$ & $8(15,1 \%)$ & 0,098 \\
\hline IAM, n (\%) & $5(10,6 \%)$ & $4(7,5 \%)$ & 0,731 \\
\hline Diálise, n (\%) & $9(19,1 \%)$ & $10(18,9 \%)$ & 0,971 \\
\hline LRA, n (\%) & $12(25,5 \%)$ & $17(32,1 \%)$ & 0,472 \\
\hline
\end{tabular}

Legenda: * Teste qui Quadrado ou exato de FisherVM> 48h - Ventilação Mecânica por mais de 48 horas; LRA- Lesão Real Aguda; LPP- Lesão por Pressão; TIH - tempo de internação hospitalar; TUTI - tempo de internação em UTI; AVE - Acidente Vascular Encefálico; IAM - Infarto Agudo do Miocárdio.

Fonte: Anjos YYF, et al., 2020. 
O escore SOFA para análise foi dividido entre dois grupos, menor e maior ou igual a três, havendo predominância SOFA $<3(n=56)$ e em ambos infecção apresentou maior pontuação, sendo no grupo $<3$ $(35,7 \%)$ e $\geq 3(61,4 \%)$. Apresentou número expressivo também a VM $>48 \mathrm{~h}$ em pacientes com $\mathrm{SOFA} \geq 3$ $(59,1 \%, n=26)$. Levando em consideração patologias, a LRA mostrou maior porcentagem entre os grupos SOFA $<3(21,4 \%)$ e SOFA $\geq 3(38,6 \%)$, havendo TIH considerável com DP de $20 \pm 17$ (SOFA <3) e $29 \pm 24$ (SOFA $\geq 3$ ). De acordo com a análise do SOFA (Tabela 4) com os desfechos, houve significância estatística com a infecção $(p=0,011)$, TIH $(p=0,034), V M>48 h(p=0,000)$, LPP $(p=0,036)$, TUTI $(p=0,002)$, Diálise $(p=$ $0,062)$ e LRA $(p=0,060)$.

Tabela 4 - Análise da associação dos desfechos dos pacientes com SOFA, Brasil, 2019.

\begin{tabular}{|c|c|c|c|}
\hline Desfechos & $\begin{array}{c}\text { SOFA }<3 \\
(n=56)\end{array}$ & $\begin{array}{c}\text { SOFA } \geq 3 \\
(n=44)\end{array}$ & $p$ valor \\
\hline Infecção, n (\%) & $20(35,7 \%)$ & $27(61,4 \%)$ & $0,011^{*}$ \\
\hline $\mathrm{TIH}$, dias, média $\pm \mathrm{DP}$ & $20 \pm 17$ & $29 \pm 24$ & $0,034^{*}$ \\
\hline VM> 48h, n (\%) & $11(19,6 \%)$ & $26(59,1 \%)$ & $0,000^{*}$ \\
\hline Morte, n (\%) & $12(21,4 \%)$ & $15(34,1 \%)$ & 0,151 \\
\hline LPP, n (\%) & $9(16,1 \%)$ & $15(34,1 \%)$ & $0,036^{*}$ \\
\hline TUTI, dias, média \pm DP & $9 \pm 8$ & $18 \pm 19$ & $0,002^{*}$ \\
\hline Reinternação, n (\%) & $6(10,7 \%)$ & $8(18,2 \%)$ & 0,285 \\
\hline AVE, n (\%) & $7(12,5 \%)$ & $3(6,8 \%)$ & 0,506 \\
\hline IAM, n (\%) & $5(8,9 \%)$ & $4(9,1 \%)$ & 1,000 \\
\hline Diálise, n (\%) & $7(12,5 \%)$ & $12(27,3 \%)$ & $0,062^{*}$ \\
\hline LRA, n (\%) & $12(21,4 \%)$ & $17(38,6 \%)$ & $0,060^{*}$ \\
\hline
\end{tabular}

Legenda: * Teste qui Quadrado ou exato de FisherVM> 48h - Ventilação Mecânica por mais de 48 horas; LRA- Lesão Real Aguda; LPP- Lesão por Pressão; TIH - tempo de internação hospitalar; TUTI - tempo de internação em UTI; AVE - Acidente Vascular Encefálico; IAM - Infarto Agudo do Miocárdio.

Fonte: Anjos YYF, et al., 2020.

O ICC foi segregado em menor e maior ou igual a quatro. Foi possível observar que seguiu o mesmo padrão do SOFA, no sentido de infecção ter apresentado maior porcentagem, < $4(31,4 \%)$ e $\geq 4(55,4 \%)$. Analisando a associação do ICC com os desfechos, houve significância estatística com infecção $(p=0,022)$, morte $(p=0,002)$, LPP $(p=0,025)$ e AVE $(p=0,013)$. A Tabela 5 traz a análise da associação dos desfechos dos pacientes com ICC.

Tabela 5 - Análise da associação dos desfechos dos pacientes com ICC, Brasil, 2019

\begin{tabular}{|c|c|c|c|}
\hline Desfechos & $\begin{array}{l}I C C<4 \\
(n=35)\end{array}$ & $\begin{array}{l}\text { ICC } \geq 4 \\
(n=65)\end{array}$ & $p$ valor \\
\hline Infecção, n (\%) & $11(31,4 \%)$ & $36(55,4 \%)$ & $0,022^{*}$ \\
\hline TUTI, dias, média \pm DP & $10 \pm 13$ & $14 \pm 15$ & 0,185 \\
\hline$V M>48 h, n(\%)$ & $9(25,7 \%)$ & $28(43,1 \%)$ & 0,086 \\
\hline Morte, n (\%) & $3(8,6 \%)$ & $24(36,9 \%)$ & $0,002^{*}$ \\
\hline LPP, n (\%) & $4(11,4 \%)$ & $20(30,8 \%)$ & $0,025^{*}$ \\
\hline TIH, dias, média \pm DP & $19 \pm 17$ & $26 \pm 22$ & 0,113 \\
\hline Reinternação, n (\%) & $2(5,7 \%)$ & $12(18,5 \%)$ & 0,129 \\
\hline AVE, n (\%) & $0(0 \%)$ & $10(15,4 \%)$ & $0,013^{*}$ \\
\hline IAM, n (\%) & $4(11,4 \%)$ & $5(7,7 \%)$ & 0,533 \\
\hline Diálise, $\mathrm{n}(\%)$ & $5(14,3 \%)$ & $14(21,5 \%)$ & 0,378 \\
\hline LRA, n (\%) & $6(17,1 \%)$ & $23(35,4 \%)$ & 0,055 \\
\hline
\end{tabular}

Legenda: * Teste qui Quadrado ou exato de FisherVM> 48h - Ventilação Mecânica por mais de 48 horas; LRA- Lesão Real Aguda; LPP- Lesão por Pressão; TIH - tempo de internação hospitalar; TUTI - tempo de internação em UTI; AVE - Acidente Vascular Encefálico; IAM - Infarto Agudo do Miocárdio.

Fonte: Anjos YYF, et al., 2020. 


\section{DISCUSSÃO}

A literatura europeia e norte-americana traz que há prevalência de pacientes do sexo feminino nas admissões na UTI em 73\% e 69\%, respectivamente (OSTAPOWICZ G, et al., 2002; REUBEN A, et al., 2016). Em estudos realizados no Brasil, mostram que há predominância de internação dos pacientes do sexo masculino e que a razão para este grupo ser o maior alvo são os hábitos que possuem, como tabagismo, etilismo e baixa adesão à prevenção e promoção a saúde (RODRIGUEZ AH, et al., 2016; NOGUEIRA LS, et al., 2012).

É possível observar que há divergência entre a literatura brasileira, europeia e norte-americana quando se trata do sexo dos pacientes admitidos na UTI. Neste estudo, apresenta-se equivalência entre os sexos da população acima de 18 anos. Quanto à idade, há variação.

O estudo realizado em um hospital escola do Paraná e Recife apresentam como idade média a faixa etária predominante entre 40 e 50 anos e no presente estudo foi $68 \pm 19$ anos, mostrando que os pacientes admitidos na referida UTI estão acima da média da idade dos outros estudos observados (HISSA PNG, et al., 2013; CAMUCI MB, et al., 2014).

A procedência da maior parte dos pacientes deste estudo foi à emergência (74\%), o que corrobora com alguns artigos observados que trazem que os pacientes admitidos na UTI são provenientes da emergência (pronto atendimento), visto que é o setor direcionado aos casos agudos, mesmo que haja uma comorbidade base (RODRIGUEZ AH, et al., 2016; NOGUEIRA LS, et al., 2012; CAMUCI MB, et al., 2014; FAVARIN SS, e CAMPONOGARA S, 2012).

Em um estudo epidemiológico, descritivo e retrospectivo realizado em uma UTI adulto da cidade de Florianópolis, o motivo mais frequente de internação foi relacionado ao sistema cardiovascular $(23,3 \%$, ) que mostra equivalência com o presente estudo, onde apresentou 31\% ( $n=29)$ (CALDEIRA VMH, et al., 2010).

Em um estudo realizado em UTI do hospital de Campinas/SP, foram avaliados como antecedentes pessoais: HAS, DM, DPOC, Tabagismo, alcoolismo, insuficiência hepática e renal e câncer, mas não expôs qual destes apresentou maior prevalência. É possível notar que alguns tópicos que foram elencados como antecedentes pessoais, no presente estudo foram enquadrados como comorbidade. Sendo assim, como principal antecedente pessoal encontrado foi cirurgia prévia (44\%), seguido de IAM prévio (15\%), tabagista prévio (12\%), AVE prévio (9\%) e Creatinina basal > 1,5 (9\%) (OLIVEIRA ABD, et al., 2010).

Quanto às comorbidades, a HAS apresentou maior prevalência nesse estudo (58\%). Com o avançar da idade ocorre surgimento de comorbidades, que aumentam a permanência hospitalar e taxa de mortalidade e a HAS é a doença vascular que mais acomete a população brasileira (LADEIA AM e LIMA BGC, 2014; LIMA DA e CEZARIO VOB, 2014).

Em estudo realizado em um hospital em João Pessoa mostrou que a grande maioria foi submetida à SVD como procedimento invasivo (86,5\%), seguido de CVC (66,8\%), SNG (65,5\%), VM (52,5\%) e TQT (10,7\%) (FIGUEIREDO DA, et al., 2013). Nesse estudo houve prevalência de SVD (35\%), seguido de CVC (29\%), o que corrobora com o estudo realizado no hospital de João Pessoa.

Escores foram aplicados no momento da alta e diariamente até o desfecho. Foram obtidos resultados dos escores admissionais ICC $4 \pm 3$, SAPS III $28 \pm 12$, SOFA $3 \pm 3$ e no desfecho SOFA $2 \pm 4$ e SAPS III $29 \pm 16$. Em estudo realizado em uma UTI da cidade de Recife, os pacientes que foram a óbito apresentaram SOFA 8 , e os que evoluíram para alta hospitalar, 4 pontos (HISSA PNG et al., 2013). Em outro estudo em hospital do sul do país, os pacientes que obtiveram pontuação de SOFA $\geq 12$ possuíram sobrevida menor (RODRIGUES FILHO EM, et al., 2018).

Em estudo realizado em hospital de João Pessoa mostrou que o maior sítio de infecção é a corrente sanguínea $(38,59 \%)$, em seguida foco pulmonar $(36,84 \%)$, trato urinário $(21,05 \%)$, cirúrgico $(3,51 \%)$ (FIGUEIREDO DA, et al., 2013). Há divergência nos resultados desse estudo citado e o presente, em que os pacientes apresentaram predominância de foco pulmonar (43\%). 
Em uma pesquisa prospectiva em UTI adulto de Campinas, observou que 52,4\% necessitaram de ventilação mecânica invasiva por tempo maior de 24 horas e $47,6 \%<24$ horas. Quanto ao presente estudo, foi utilizado o parâmetro de VM > 48 horas onde se fez necessário em 37\% (OLIVEIRA ABD, et al., 2010).

Nogueira LS, et al. (2012) relatam que LRA é mais frequente, estando presente em $69,68 \%$ dos pacientes, onde alguns necessitam de diálise. Quanto à LPP, 22,2\% a 41,2\% dos pacientes desenvolvem na UTI (ROGENSKY NMB e KURCGANT P, 2012; PEREIRA LC, et al., 2013; SOUSA PRA, et al., 2013).

No estudo de Rodriguez e outros autores (RODRIGUEZ AH, et al., 2016), o óbito foi desfecho de $20,4 \%$ dos pacientes. Levando em conta esses pontos, esse estudo apresentou que $29 \%$ desenvolveram LRA, e $19 \%$ precisaram de diálise, 24\% apresentaram LPP em decorrência da internação, $27 \%$ da amostra foi a óbito, e o desfecho com menor porcentagem foi o IAM apresentando $9 \%$.

Em dois estudos realizados em um hospital do Rio Grande do Sul e São Paulo mostraram que o tempo de permanência na UTI foi de 9 a 16 dias e tempo de internação hospitalar médio de 14 dias (NOGUEIRA LS, et al., 2012). No presente estudo, a média do tempo de internação hospitalar (TIH) foi 16 e tempo em unidade de terapia intensiva (TUTI) 8.

De acordo com estudo feito em duas UTIs no estado de São Paulo, o SAPS III apresentou média de $48,5 \pm 18,1$. Diferentemente do estudo presente, as operações gastrintestinais foram predominantes $(34,9 \%)$, seguidas das ortopédicas (28,2\%) (SILVA JÚNIOR JM, et al., 2010). Há divergência nos resultados desse estudo citado e o presente, tendo predominância com a infecção ( $<28=34 \%$ ) e ( $>$ ou igual $28=66 \%$ ).

Em um estudo na UTI do Hospital Regional de Patos, tendo o resultado de $46,875 \%$ de morte. Mais uma divergência com o estudo presente, que foi de apenas $21,3 \%$ com SAPS III $<28$ e $32,1 \%$ com SAPSIII $>$ ou igual 28 (TIMÓTEO PAD, et al., 2018).

Estudo prospectivo realizado em uma UTI de adultos clínica/cirúrgica do Hospital Agamenon Magalhães, os pacientes que foram a óbito (75\%) apresentaram SOFA 8 (HISSA PNG, et al., 2013). Já no estudo presente, o desfecho de morte com SOFA $<3$ foi $21,4 \%$ e SOFA $>$ ou igual a 3 foi $34,1 \%$. Mostrando que quanto maior o SOFA, maior a chance de morte na UTI.

Fernandes SEF, et al. (2014) realizaram estudo em UTI de Santa Rita-PB, onde obtiveram o resultado de que o maior desfecho foi pneumonia (60\%), utilizando o SOFA 12 e 13, sendo $p=1,0$. No estudo presente, $o$ maior desfecho foi infecção, SOFA $<3(35,7 \%)$ e SOFA > ou igual a $3(61,4 \%)$, sendo $p=0,011$.

De acordo com estudo realizado a partir de informações do Sistema de Informação Hospitalar do Sistema Único de Saúde (SIH/SUS), observou-se que o IAM possui 11,7\% (MARTINS M, 2010). No estudo presente, houve uma equivalência, aonde IAM com ICC < 4 obteve $11,4 \%$. Segundo dados de estudos realizados na UTI do Hospital Regional de Paraná, o desfecho de melhora obteve maior porcentagem (53,5\%), posteriormente têm a morte (38,6\%) e por último a transferência, com apenas 7,9\% (GUIA CM, et al., 2015). Já no presente estudo e de acordo com a tabela anterior, o maior desfecho foi a infecção com ICC $\geq 4(55,4 \%)$, seguida de TUTI com ICC $\geq 4$ (14 \pm 15$)$ e por último, LRA com ICC $\geq 4(35,4 \%)$.

Ao aplicar os escores no momento da admissão e desfecho é possível correlacionar se houve melhora no quadro clínico e auxiliar a equipe multidisciplinar para traçar uma assistência direcionada às necessidades, apresentando um ponto positivo para o paciente. Através dos resultados obtidos é possível observar que ao saber o perfil dos pacientes admitidos na UTI do hospital onde foi realizada a pesquisa, toda equipe consegue ter parâmetro da qualidade da assistência prestada e se há desenvolvimento de infecções, ou complicações inerentes ao internamento no setor. Após avaliação da literatura, alguns dados corroboraram com a pesquisa, outros apresentaram divergência, entretanto vale ressaltar que apesar de comparar o estudo com diversos, existem particularidades como o perfil institucional, particular ou privada, característica da UTI, mista, clínica ou cirúrgica, e perfil da população que é assistida.

Por fim, pode-se evidenciar a associação de desfechos clínicos desfavoráveis ao paciente com escores assistências. Tornando-se essencial sua utilização na prática clínica de modo a guiar a assistência prestada ao paciente crítico. 


\section{CONCLUSÃO}

A procedência prevalente foi à emergência, o diagnóstico por sistema foi o cardiovascular que apresentou maior porcentagem, antecedentes pessoais cirurgia prévia, HAS como comorbidade, suporte admissional noradrenalina e fentanil, dispositivo mais utilizado SVD. Quanto ao grupo de maior prevalência entre os escores, SAPSIII $\geq 28$, SOFA $<3$ e ICC $\geq 4$ e infecção mostrou predominância na avaliação do desfecho. Foi possível avaliar a associação entre a admissão e o desfecho dos pacientes, sendo observado quanto maior o SOFA, menor a chance de sobrevida.

\section{REFERÊNCIAS}

1. AUSTIN SR, et al. Why summary comorbidity measures such as the Charlson Comorbidity Index and Elixhauser score work. Medical Care 2015; 53(9): 65-72.

2. BRASIL. Média de permanência UTI adulto. Ministério da Saúde 2013; 1(1).

3. BUENO HL, BIATTO JFP. Epidemiologia e validação de escore prognóstico em UTI mista no norte do Paraná. Revista UNINGÁ Review 2015; 22(3): 23-29.

4. CALDEIRA VMH, et al. Critérios para admissão de pacientes na Unidade de Terapia Intensiva e mortalidade. Revista da Associação Médica Brasileira 2010; 56(5): 528-534.

5. CAMUCI MB, et al. Nursing Activities Score: carga de trabalho de enfermagem em Unidade de Terapia Intensiva de queimados. Revista Latino-Americana de Enfermagem 2014; 22(2): 325-331.

6. FAVARIN SS, CAMPONOGARA S. Perfil dos pacientes internados na Unidade de Terapia Intensiva adulto de um Hospital Universitário. Revista de Enfermagem da UFSM 2012; 2(2): 320-329.

7. FERNANDES SEF, et al. Avaliação da mortalidade na unidade de terapia intensiva do hospital Governador Flávio Ribeiro em Santa Rita-PB (HGFRC), segundo escore SOFA. Revista Ciência Saúde Nova Esperança 2014; 12(1): $15-21$.

8. FIGUEIREDO DA, et al. Epidemiologia da Infecção Hospitalar em uma Unidade de Terapia Intensiva de um Hospital Público Municipal de João Pessoa-PB. Revista Brasileira de Ciências da Saúde 2013; 17(3): 233-240.

9. GUIA CM, et al. Perfil epidemiológico e preditores de mortalidade de uma unidade de terapia intensiva geral de hospital público do Distrito Federal. Comunicação em Ciências da Saúde 2015; 26(1/2): 9-19.

10. HISSA PNG, et al. Análise comparativa entre dois escores na previsão de mortalidade em unidade de terapia intensiva. Revista Brasileira de Clínica Médica 2013; 11(1): 21-26.

11. KEEGAN MT, SOARES M. O que todo intensivista deveria saber o sistema de escore prognóstico e mortalidade ajustada ao risco. Revista Brasileira de Terapia Intensiva 2016; 28(3): 264-269.

12. LADEIA AM, LIMA BGC. Hipertensão arterial sistêmica e comorbidades associadas: relevância epidemiológica do acidente vascular cerebral no Brasil. Sociedade Brasileira de Hipertensão 2014; 17(3-4): 156-162.

13. LIMA DA, CEZARIO VOB. Quedas em idosos e comorbidades clínicas. Revista HUPE 2014; 13(2): 30-37.

14. MARTINS M. Uso de medidas de comorbidades para predição de risco de óbito em pacientes brasileiros hospitalizados. Revista de Saúde Pública 2010; 44(33): 448-458.

15. NOGUEIRA LS, et al. Características clínicas e gravidade de pacientes internados em UTIs públicas e privadas. Texto e Contexto Enfermagem 2012; 21(1): 59-67.

16. OSTAPOWICZ G, et al. Results of a prospective study of acute liver failure at 17 tertiary care centers in the United States. Annals of Internal Medicine 2002; 137(12): 947-954.

17. OLIVEIRA ABD, et al. Fatores associados à maior mortalidade e tempo de internação prolongado em uma unidade de terapia intensiva de adultos. Revista Brasileira de Terapia Intensiva 2010; 22(3): 250-256.

18. PASSOS SSS, ET al. O acolhimento no cuidado à família numa Unidade de Terapia Intensiva. Revista de Enfermagem UFRJ 2015; 23(3): 368-374.

19. PEREIRA LC, et al. Incidência de úlceras por pressão em uma unidade de terapia intensiva de um hospital público. Revista de Enfermagem da UFPI 2013; 2(4): 21-27.

20. REUBEN A, et al. Outcomes in Adults with Acute Liver Failure Between 1998 and 2013: Na Observational Cohort Study. Annals of Internal Medicine 2016;164(11): 725-732.

21. RODRIGUES FILHO EM, et al. SOFA nas primeiras 24 horas como preditor de desfecho em insuficiência hepática aguda. Revista Brasileira de Terapia Intensiva 2018, 30(1): 64-70.

22. RODRIGUEZ AH, et al. Características epidemiológicas e causas de óbitos em pacientes internados em terapia intensiva. Revista Brasileira de Enfermagem 2016; 69(2): 229-234.

23. ROGENSKY NMB, KURCGANTE P. Incidência de úlceras por pressão após a implementação de um protocolo de prevenção. Revista Latino-Americana de Enfermagem 2012; 20(2): 07 telas.

24. SILVA JÚNIOR JM, et al. Aplicabilidade do escore fisiológico agudo simplificado (SAPS 3) em hospitais brasileiros. Revista Brasileira de Anestesiologia 2010; 60(1): 20-31.

25. SILVA LM, et al. Índices prognósticos na prática clínica de enfermagem em terapia intensiva: revisão integrativa. Revista Eletrônica de Enfermagem 2014; 16(1): 179-190.

26. SOUSA PRA, et al. Avaliação de risco para desenvolvimento de Úlceras por Pressão em pacientes críticos. Revista de Enfermagem da UFPI 2013; 2(1): 9-15

27. TIMÓTEO PAD, et al. Avaliação de índices prognósticos preditivos de mortalidade dos pacientes admitidos em terapia intensiva. Journal of medicine and health promotion 2018; 3(1): 935-945. 